\title{
THE KP HiERARCHY In Miwa COORDinates *
}

\author{
Boris Konopelchenko \\ Dipartimento di Fisica, Universitá de Lecce \\ 73100 Lecce, Italy \\ Luis Martínez Alonso \\ Departamento de Física Teórica, Universidad Complutense \\ E28040 Madrid, Spain
}

\begin{abstract}
A systematic reformulation of the KP hierarchy by using continuous Miwa variables is presented. Basic quantities and relations are defined and determinantal expressions for Fay's identities are obtained. It is shown that in terms of these variables the KP hierarchy gives rise to a Darboux system describing an infinite-dimensional conjugate net.
\end{abstract}

Key words: KP hierarchy, tau-functions, Miwa variables. 1991 MSC: 58B20.

*Partially supported by CICYT proyecto PB95-0401 


\section{Introduction}

The Kadomtsev-Petviashvili(KP) equation, introduced to describe propagation of shallow water waves under special conditions [1], is nowadays a principal ingredient in studies of many problems in physics and mathematics. The KP hierarchy is a paradigm of the hierarchies of integrable systems [2]- [3], it is used to characterize Jacobian varieties in algebraic geometry [4] and is connected to string theory due to the close relation arising between partition functions of quantum models and KP $\tau$-functions [5, 77.

The standard variables for the KP hierarchy form an infinite-dimensional vector $\boldsymbol{t}=\left(t_{1}, t_{2}, \ldots\right)$, the three first components of which being the two spatial and one time variables of the KP equation viewed as a hydrodinamical model. The KP hierarchy can be formulated as the compatibility conditions for the following linear system of equations (see e.g. [2, 3, 9, 10])

$$
\partial_{n} \psi=P_{n}\left(\boldsymbol{t}, \partial_{x}\right) \psi, \quad \partial_{n}=\frac{\partial}{\partial t_{n}}, n \geq 2 .
$$

Here $P_{n}\left(\boldsymbol{t}, \partial_{x}\right)$ denotes a set of linear differential operators with respect to the variable $x \equiv t_{1}$ and $\psi=\psi(z, \boldsymbol{t})$ is the $\mathrm{KP}$ wave function, a complex-valued function defined on the unit circle $(|z|=1)$ which admits a factorization $\psi=\psi_{0} \chi$, where

$$
\psi_{0}=\exp \left(\sum_{n \geq 1} \frac{t_{n}}{z^{n}}\right), \quad \chi=1+\sum_{n \geq 1} a_{n}(\boldsymbol{t}) z^{n} .
$$

Another remarkable parametrization of the KP hierarchy is provided by the so-called Miwa variables defined as

$$
t_{n}=\frac{1}{n} \sum_{i=1}^{\infty} p_{i} z_{i}^{n}, \quad n \geq 1,
$$

where $p_{i}$ and $z_{i}$ are discrete (integer valued) and continuous (complex valued) Miwa variables, respectively. It is worth-mentioning that the KP hierarchy can be derived from a single finite-difference Hirota equation involving the discrete Miwa variables [1] , which in turn is closely connected with the Fay's trisecant formula.

Miwa variables have been extensively used several years ago in the study of matrix models in string theory and two-dimensional quantum gravity [5][7]. In particular, they were relevant to show that partition functions for 
matrix models of Kontsevich type are connected with KP $\tau$-functions. On the other hand, in a different approach to string theory via the KP hierarchy it was found that Miwa variables $p_{i}$ and $z_{i}$ can be identified with the momenta and Koba-Nielsen variables of strings [12]. At last, it was recently shown 13 that Miwa variables are also useful in the analysis of the Bethe ansatz for quantum integrable systems.

From the point of view of mathematics, sets of variables of the types $t_{n}$ and $z_{i}$ connected by (3) have been used for a long time in the representation theory of the symmetric group $S_{N}[14,15,16]$. In this context $t_{n}$ and $z_{i}$ are referred to as power sums and symmetric variables, respectively. The presence of these variables in the theory of the KP hierarchy is a consequence of the well established relationship between this hierarchy and the representation theory of loop groups [16]-17].

A main goal of the present paper is to reformulate the KP hierarchy, its basic objects and relations, using symmetric ( continuous) Miwa variables. These variables and the spectral parameters appear on an equal footing in the expressions for $\tau$-functions and wave functions. Thus, it follows that several fundamental relations for the KP hierarchy as, for instance, Sato's equations, the relationship between wave functions and $\tau$-functions and the action of Darboux-Bäcklund transformations take a symmetric and simple form. Furthermore, Miwa variables are very useful to deal with the bilinear identity for $\tau$-functions. In particular, we prove that they provide a compact determinantal form for addition formulae (Fay's identities). We also prove that the simplest Fay's identity, written in terms of Miwa variables, leads to a Darboux system describing an infinite-dimensional conjugate net.

\section{KP flows and $\tau$-functions}

The KP hierarchy of integrable systems can be introduced by means of the Sato's flows [8, 9, 4]

$$
\frac{\partial S}{\partial t_{n}}=-\left(S \partial_{x}^{n} S^{-1}\right)_{-} S
$$

for the pseudo-differential operator

$$
S:=1+\sum_{n \geq 1} a_{n}(\boldsymbol{t}) \partial_{x}^{-n}
$$


The solutions of (45) can be related to KP wave functions by setting the same coefficients $a_{n}(\boldsymbol{t})$ in the expansions (2) of $\chi$ and (5) of $S$.

On the other hand, it is well known [9], 16] that a KP wave function leads to a flow in an infinite-dimensional Grassmannian. To describe this property we consider the Hilbert space $H:=L^{2}\left(S^{1}, \mathbb{C}\right)$ of square-integrable complex-valued functions on the unit circle $S^{1}$ and the big cell $\mathrm{Gr}_{0}$ of the Grassmannian Gr of $H$. For our purposes $\mathrm{Gr}_{0}$ can be defined as the set of all closed subspaces $W$ of $H$ which admit a dense linear subspace generated by an admissible basis; i.e. a subset $\left\{w_{n}=w_{n}(z) n \geq 0\right\} \subset W$ of finite order elements:

$$
w_{n}(z)=z^{-n}+\mathcal{O}\left(z^{-n+1}\right) .
$$

Each KP wave function $\psi$ determines an element $W$ of $\mathrm{Gr}$ defined as the closure in $H$ of the set of all linear combinations of the form

$$
\sum_{n \geq 1}^{N} c_{n}\left(\boldsymbol{t}_{n}\right) \psi\left(z, \boldsymbol{t}_{n}\right) .
$$

Here $c_{n}$ are arbitrary functions of $\boldsymbol{t}$ and $\boldsymbol{t}_{n}$ are arbitrary points of the domain of $\psi$ in $\mathbb{C}^{\infty}$. From (11) and by using Taylor expansion around any fixed $\boldsymbol{t}$ it follows that

$$
W=\operatorname{span}\left\{\partial_{x}^{n} \psi(z, \boldsymbol{t}), n \geq 0, \text { any fixed } \boldsymbol{t}\right\},
$$

Thus each KP wave function determines a flow in Gr given by

$$
W(\boldsymbol{t}):=\psi_{0}(z, \boldsymbol{t})^{-1} W=\operatorname{span}\left\{\psi_{0}(z, \boldsymbol{t})^{-1} \partial_{x}^{n} \psi(z, \boldsymbol{t}), n \geq 0,\right\} .
$$

For all value of $\boldsymbol{t}$ in the domain of $\psi$ in $\mathbb{C}^{\infty}$ we have that

$$
\psi_{0}(z, \boldsymbol{t})^{-1} \partial_{x}^{n} \psi(z, \boldsymbol{t})=z^{-n}+\mathcal{O}\left(z^{-n+1}\right),
$$

so that $W(\boldsymbol{t}) \in \mathrm{Gr}_{0}$.

There is a natural imbeding of $\mathrm{Gr}$ in the projective space $P(\mathcal{H})$ of the infinite wedge space $\mathcal{H}:=\wedge^{\infty} H$. It assigns to each $W \in$ Gr the ray $|W\rangle$ in $\mathcal{H}$ generated by the vectors

$$
w_{0} \wedge w_{1} \wedge \cdots \wedge w_{n} \wedge \cdots
$$

where $\left\{w_{n}\right\}_{n \geq 0}$ is any admissible basis of $W$. 
A set of fermionic operators on $\mathcal{H}$ can be defined by

$$
b_{n}=\frac{\partial}{\partial e_{n}}, \quad c_{n}=e_{n} \wedge \cdot, n=0, \pm 1, \pm 2, \ldots,
$$

where $e_{n}:=z_{-n}$, the operator $\frac{\partial}{\partial e_{n}}$ denotes the supersymmetric derivative with respect to $e_{n}$ and the operator $e_{n} \wedge$. is the exterior multiplication operator by $e_{n}$. They satisfy the canonical anticommutation relations

$$
\left\{b_{n}, b_{m}\right\}=\left\{c_{n}, c_{m}\right\}=0, \quad\left\{b_{n}, c_{m}\right\}=\delta_{n m} .
$$

Moreover, if we define the vacuum state as

$$
|v a c\rangle:=|0\rangle=e_{0} \wedge e_{1} \wedge e_{2} \wedge \ldots \wedge e_{n} \wedge \ldots,
$$

it follows that

$$
b_{n}|v a c\rangle=0,(n<0) ; \quad c_{n}|v a c\rangle=0,(n \geq 0) .
$$

These fermionic modes determine the following fields on the unit circle

$$
b(z):=\sum_{n} b_{n} z^{-n}, \quad c(z):=\sum_{n} c_{n} z^{n}, \quad|z|=1 .
$$

It can be shown [9] that given a $\mathrm{KP}$ wave function $\psi=\psi_{0} \chi$ we may write

$$
\chi(z, \boldsymbol{t})=\frac{\tau(\boldsymbol{t}-[z])}{\tau(\boldsymbol{t})},[z]:=\left(z, \frac{z^{2}}{2}, \frac{z^{3}}{3}, \ldots\right) .
$$

Here the function $\tau$ is defined up to a multiplicative constant by the correlation function

$$
\tau(\boldsymbol{t}):=\langle\operatorname{vac} \mid W(\boldsymbol{t})\rangle
$$

where $W(\boldsymbol{t})$ is the KP trajectory of the element $W \in G r_{0}$ generated by $\psi$. Moreover, it turns out [9] that

$$
|W(\boldsymbol{t})\rangle=\exp (H(\boldsymbol{t}))|W\rangle, \quad H(\boldsymbol{t}):=\sum_{n \geq 1} t_{n}\left(\sum_{m=-\infty}^{\infty} b_{m} c_{m+n}\right) .
$$

The following bilinear identity constitutes the main property of $\tau$-functions

$$
\int_{S^{1}} \exp \left(\sum_{n \geq 1} z^{-n}\left(t_{n}-t_{n}^{\prime}\right)\right) \tau(\boldsymbol{t}-[z]) \tau\left(\boldsymbol{t}^{\prime}+[z]\right) \frac{d z}{z^{2}}=0 .
$$


Natural symmetries of this identity are the so called Darboux-Bäcklund transformations [18

$$
\mathcal{D}(p, \boldsymbol{t}) \tau(\boldsymbol{t}):=\psi_{0}(p, \boldsymbol{t}) \tau(\boldsymbol{t}-[p])
$$

and their dual analogues

$$
\mathcal{D}^{*}(p, \boldsymbol{t}) \tau(\boldsymbol{t}):=\psi_{0}(p, \boldsymbol{t})^{-1} \tau(\boldsymbol{t}+[p]) .
$$

As a consequence of (11) every KP wave function $\psi$ has an associated adjoint function $\psi^{*}=\left(\psi_{0}\right)^{-1} \chi^{*}$ with

$$
\chi^{*}(z, \boldsymbol{t})=\frac{\tau(\boldsymbol{t}+[z])}{\tau(\boldsymbol{t})},
$$

which verifies the bilinear identity

$$
\int_{S^{1}} \psi(z, \boldsymbol{t}) \psi^{*}\left(z, \boldsymbol{t}^{\prime}\right) \frac{d z}{z^{2}}=0 .
$$

From (11) it also follows that

$$
\int_{S^{1}} \psi(z, u, \boldsymbol{t}) \psi\left(u^{\prime}, z, \boldsymbol{t}^{\prime}\right) d z=0,
$$

where

$$
\psi\left(z, z^{\prime}, \boldsymbol{t}\right)=\psi_{0}(z, \boldsymbol{t}) \chi\left(z, z^{\prime}, \boldsymbol{t}\right) \psi_{0}^{-1}\left(z^{\prime}, \boldsymbol{t}\right)
$$

is the Cauchy-Baker function introduced in [19] which is determined by

$$
\chi\left(z, z^{\prime}, \boldsymbol{t}\right):=\frac{1}{z-z^{\prime}} \frac{\tau\left(\boldsymbol{t}-[z]+\left[z^{\prime}\right]\right)}{\tau(\boldsymbol{t})} .
$$

\section{Miwa variables}

We introduce Miwa variables $\boldsymbol{x}=\left(x_{1}, x_{2}, x_{3}, \ldots\right)$ and $\boldsymbol{y}=\left(y_{1}, y_{2}, y_{3}, \ldots\right)$ $\left|x_{i}\right|,\left|y_{i}\right|<1$ by

$$
\boldsymbol{t}=\sum_{i=1}^{\infty}\left(\left[x_{i}\right]-\left[y_{i}\right]\right)
$$


or equivalently

$$
t_{n}=\frac{1}{n}\left(\sum_{i=1}^{\infty}\left(x_{i}^{n}-y_{i}^{n}\right)\right), \quad n \geq 1 .
$$

Our definition, slightly more general than those used in [11] or [14, 15, 16], is also adopted in [6, 19, 20]. Transformations of the type (18) are useful in the theory of symmetric polynomials [14, 15]. To show this relationship we just mention that the Jacobian matrix of the transformation (18) is given by

$$
\begin{aligned}
\frac{\partial x_{i}}{\partial t_{n}} & =-(-1)^{n} \frac{\phi_{n-1}\left(x_{1}, \ldots, \not_{i}, \ldots ; y_{1}, y_{2} \ldots\right)}{\prod_{m \neq i}\left(1-x_{i} / x_{m}\right) \prod_{n}\left(1-x_{i} / y_{m}\right)}, \\
\frac{\partial y_{i}}{\partial t_{n}} & =(-1)^{n} \frac{\phi_{n-1}\left(x_{1}, x_{2}, \ldots, ; y_{1}, \ldots, y_{i}, \ldots\right)}{\prod_{m \neq i}\left(1-y_{i} / y_{m}\right) \prod_{m}\left(1-y_{i} / x_{m}\right)},
\end{aligned}
$$

where $\phi_{n}$ are the symmetric functions

$$
\phi_{n}\left(z_{1}, z_{2} \ldots\right)=\sum_{i_{1}<i_{2}<\ldots<i_{n}} \frac{1}{z_{i_{1}}} \frac{1}{z_{i_{2}}} \ldots \frac{1}{z_{i_{n}}} .
$$

These expressions can be derived by using the following standard identity of the theory of symmetric polynomials

$$
\prod_{n \geq 1}\left(1-\frac{\lambda}{z_{n}}\right)=\sum_{n \geq 0}(-\lambda)^{n} \phi_{n}\left(z_{1}, z_{2}, \ldots\right),
$$

and taking into account that

$$
\frac{\partial t_{n}}{\partial x_{i}}=x_{i}^{n-1}, \frac{\partial t_{n}}{\partial y_{i}}=-y_{i}^{n-1} .
$$

Given a function $\alpha(\boldsymbol{t})$ depending on the standard KP variables we will denote by $\alpha(\boldsymbol{x} ; \boldsymbol{y})$ the corresponding function depending on the Miwa variables. In what follows the following obvious properties will be useful

(1) The function $\alpha(\boldsymbol{x} ; \boldsymbol{y})$ is invariant under permutations among the components of $\boldsymbol{x}$ or $\boldsymbol{y}$.

(2) $\alpha(0, \boldsymbol{x} ; \boldsymbol{y})=\alpha(\boldsymbol{x} ; 0, \boldsymbol{y})=\alpha(\boldsymbol{x} ; \boldsymbol{y})$ 
(3) For any given finite or infinite-dimensional vector $\boldsymbol{a}=\left(a_{1}, a_{2}, \ldots\right)$

$$
\alpha(\boldsymbol{a}, \boldsymbol{x} ; \boldsymbol{a}, \boldsymbol{y})=\alpha(\boldsymbol{x} ; \boldsymbol{y}) .
$$

Let us rewrite the basic objects of the KP theory in terms of Miwa variables. First of all we notice that (4) and (19) imply that Sato's flows in Miwa variables can be written as

$$
\frac{\partial S}{\partial x_{i}}=-\left(S \frac{\partial_{x}}{1-x_{i} \partial_{x}} S^{-1}\right)_{-} S, \quad \frac{\partial S}{\partial y_{i}}=\left(S \frac{\partial_{x}}{1-y_{i} \partial_{x}} S^{-1}\right)_{-} S .
$$

The vacuum wave function takes the form

$$
\psi_{0}(z ; \boldsymbol{x} ; \boldsymbol{y})=\prod_{i \geq 1} \frac{1-y_{i} / z}{1-x_{i} / z}
$$

The undressed KP wave function and its adjoint function are given by

$$
\chi(z ; \boldsymbol{x} ; \boldsymbol{y})=\frac{\tau(\boldsymbol{x} ; z, \boldsymbol{y})}{\tau(\boldsymbol{x}, \boldsymbol{y})}, \quad \chi^{*}(z ; \boldsymbol{x} ; \boldsymbol{y})=\frac{\tau(z, \boldsymbol{x} ; \boldsymbol{y})}{\tau(\boldsymbol{x}, \boldsymbol{y})} .
$$

Analogously, the Cauchy-Baker function reads

$$
\chi\left(z, z^{\prime} ; \boldsymbol{x} ; \boldsymbol{y}\right)=\frac{1}{z-z^{\prime}} \frac{\tau\left(z^{\prime}, \boldsymbol{x} ; z, \boldsymbol{y}\right)}{\tau(\boldsymbol{x} ; \boldsymbol{y})} .
$$

We would like to emphasize that the spectral parameter $z$ appears in the $\tau$-function on the equal footing as the Miwa variables. It is a $y$-type variable in the expression for the wave function, an $x$-type variable in the case of the adjoint wave function, and it takes the role of both types in the case of the Cauchy-Baker function. Thus, it is only when we introduce the wave functions we mark one of the Miwa variables of the $\tau$-function, call it spectral parameter and break its symmetry with the remaining Miwa variables of the same type. As we will discuss elsewhere, this symmetry between spectral parameters and Miwa coordinates at the $\tau$-function level is very useful for dealing with symmetries of the KP hierarchy of both standard and nonisospectral types in the same manner.

Furthermore, it can be shown [6, 19, 20] that the $\tau$-function can be expressed as

$$
\tau(\boldsymbol{x} ; \boldsymbol{y})=D(\boldsymbol{x} ; \boldsymbol{y}) \operatorname{det}\left(\frac{\left\langle v a c\left|c\left(x_{i}\right) b\left(y_{j}\right)\right| W\right\rangle}{y_{j}\langle v a c \mid W\rangle}\right)
$$


where

$$
D(\boldsymbol{x} ; \boldsymbol{y}):=\frac{\prod_{i, j}\left(y_{i}-x_{j}\right)}{\prod_{n>m}\left(y_{m}-y_{n}\right) \prod_{n>m}\left(x_{n}-x_{m}\right)} .
$$

Therefore (23) allows us to write

$$
\tau(\boldsymbol{x} ; \boldsymbol{y})=D(\boldsymbol{x} ; \boldsymbol{y}) \operatorname{det}\left(\frac{\tau\left(x_{i} ; y_{j}\right)}{y_{j}-x_{i}}\right)
$$

Thus, we see that the value of the $\tau$-function at a point $(\boldsymbol{x} ; \boldsymbol{y}) \in \boldsymbol{C}^{2 \infty}$ in Miwa space can be expressed in terms of its values at two-dimensional single points $\left(x_{i}, y_{j}\right) \in \boldsymbol{C}^{2}$. Notice also that the building block of this expression for $\tau$ is closely related to the Cauchy-Baker function as shows the identity

$$
\frac{\tau\left(x_{i} ; y_{j}\right)}{y_{j}-x_{i}}=\tau(\mathbf{0}) \chi\left(y_{j}, x_{i} ; \mathbf{0}\right) \text {. }
$$

Miwa variables are very suitable for dealing with the symmetry operations of the KP hierarchy too. For instance, let us notice that the DarbouxBäcklund transformations (12) take the form

$$
\mathcal{D}(p ; \boldsymbol{x}: \boldsymbol{y}) \tau(\boldsymbol{x} ; \boldsymbol{y})=\prod_{n \geq 1} \frac{1-y_{n} / p}{1-x_{n} / p} \tau(\boldsymbol{x} ; p, \boldsymbol{y})
$$

and

$$
\mathcal{D}^{*}(p ; \boldsymbol{x}: \boldsymbol{y}) \tau(\boldsymbol{x} ; \boldsymbol{y})=\prod_{n \geq 1} \frac{1-x_{n} / p}{1-y_{n} / p} \tau(p, \boldsymbol{x} ; \boldsymbol{y})
$$

By taking advantage of the factorized form of this expression one easily proves the usual transformation properties of the wave functions 18. For example, we have

$$
\begin{aligned}
& \mathcal{D}(p ; \boldsymbol{x}: \boldsymbol{y}) \psi(z ; \boldsymbol{x} ; \boldsymbol{y})=-\frac{z}{p} \psi(z ; \boldsymbol{x} ; p, \boldsymbol{y}), \\
& \mathcal{D}(p ; \boldsymbol{x}: \boldsymbol{y}) \psi^{*}(z ; \boldsymbol{x} ; \boldsymbol{y})=-\frac{p}{z} \psi^{*}(z ; \boldsymbol{x} ; p, \boldsymbol{y}), \\
& \mathcal{D}(p ; \boldsymbol{x}: \boldsymbol{y}) \psi\left(z, z^{\prime} ; \boldsymbol{x} ; \boldsymbol{y}\right)=-\frac{z}{z^{\prime}} \psi\left(z, z^{\prime} ; \boldsymbol{x} ; p, \boldsymbol{y}\right) .
\end{aligned}
$$




\section{Addition formulae and Darboux equations}

Let us consider the bilinear identity (11), which written in terms of Miwa variables takes the form

$$
\int_{S^{1}} \prod_{i \geq 1} \frac{\left(1-y_{i} / z\right)\left(1-x_{i}^{\prime} / z\right)}{\left(1-y_{i}^{\prime} / z\right)\left(1-x_{i} / z\right)} \tau(\boldsymbol{x} ; z, \boldsymbol{y}) \tau\left(z, \boldsymbol{x}^{\prime} ; \boldsymbol{y}^{\prime}\right) \frac{d z}{z^{2}}=0 .
$$

If we set $\boldsymbol{x} \rightarrow(\boldsymbol{s}, \boldsymbol{x}), \boldsymbol{x}^{\prime} \rightarrow\left(\boldsymbol{s}^{\prime}, \boldsymbol{x}\right), \quad \boldsymbol{y}^{\prime}=\boldsymbol{y}$, where

$$
\boldsymbol{s}=\left(s_{1}, s_{2}, \ldots, s_{N}\right), \quad \boldsymbol{s}^{\prime}=\left(s_{1}^{\prime}, s_{2}^{\prime}, \ldots, s_{M}^{\prime}\right), \quad N-M \geq 2,
$$

and all the components of $s$ and $\boldsymbol{s}^{\prime}$ are assumed to be different, then the bilinear identity becomes

$$
\int_{S^{1}} \frac{\prod_{i=1}^{M}\left(1-s_{i}^{\prime} / z\right)}{\prod_{i=1}^{N}\left(1-s_{i} / z\right)} \tau(\boldsymbol{s}, \boldsymbol{x} ; z, \boldsymbol{y}) \tau\left(z, \boldsymbol{s}^{\prime}, \boldsymbol{x} ; \boldsymbol{y}\right) \frac{d z}{z^{2}}=0 .
$$

Thus by calculating the integral as the sum of residues at $s_{i},(i=1, \ldots, N)$ we get a set of Fay's identities

$$
\sum_{i=1}^{N} \frac{\rho\left(s_{i}\right)}{\prod_{j \neq i}\left(s_{i}-s_{j}\right)} \hat{\tau}_{i} \tau_{i}=0
$$

where

$$
\rho(z):=z^{N-M-2} \prod_{i=1}^{M}\left(z-s_{i}^{\prime}\right)
$$

and

$$
\hat{\tau}_{i}:=\tau\left(s_{1}, \ldots, \$_{i}, \ldots, s_{N}, \boldsymbol{x} ; \boldsymbol{y}\right), \quad \tau_{i}:=\tau\left(s_{i}, \boldsymbol{s}^{\prime}, \boldsymbol{x} ; \boldsymbol{y}\right) .
$$

We note that these identities can be rewritten as

$$
\left|\begin{array}{ccccc}
1 & s_{1} & \ldots & s_{1}^{N-2} & \rho\left(s_{1}\right) \tau_{1} \hat{\tau}_{1} \\
1 & s_{2} & \ldots & s_{2}^{N-2} & \rho\left(s_{2}\right) \tau_{2} \hat{\tau}_{2} \\
\ldots & \ldots & \ldots & \ldots & \ldots \\
\ldots & \ldots & \ldots & \ldots & \ldots \\
1 & s_{N} & \ldots & s_{N}^{N-2} & \rho\left(s_{N}\right) \tau_{N} \hat{\tau}_{N}
\end{array}\right|=0 .
$$


The case $N=3, M=1$ is the usual Fay's identity

$$
\begin{aligned}
& \frac{s_{1}-s_{1}^{\prime}}{\left(s_{1}-s_{2}\right)\left(s_{1}-s_{3}\right)} \tau\left(s_{2}, s_{3}, \boldsymbol{x} ; \boldsymbol{y}\right) \tau\left(s_{1}, s_{1}^{\prime}, \boldsymbol{x} ; \boldsymbol{y}\right) \\
& +\frac{s_{2}-s_{1}^{\prime}}{\left(s_{2}-s_{1}\right)\left(s_{2}-s_{3}\right)} \tau\left(s_{1}, s_{3}, \boldsymbol{x} ; \boldsymbol{y}\right) \tau\left(s_{2}, s_{1}^{\prime}, \boldsymbol{x} ; \boldsymbol{y}\right) \\
& +\frac{s_{3}-s_{1}^{\prime}}{\left(s_{3}-s_{1}\right)\left(s_{3}-s_{2}\right)} \tau\left(s_{1}, s_{2}, \boldsymbol{x} ; \boldsymbol{y}\right) \tau\left(s_{3}, s_{1}^{\prime}, \boldsymbol{x} ; \boldsymbol{y}\right)=0 .
\end{aligned}
$$

By setting $\boldsymbol{y} \rightarrow\left(s_{2}, \boldsymbol{y}\right)$ and dividing by

$$
\tau\left(s_{1}^{\prime}, \boldsymbol{x} ; \boldsymbol{y}\right) \tau\left(s_{3}, \boldsymbol{x} ; \boldsymbol{y}\right),
$$

we get

$$
\begin{aligned}
& \frac{s_{1}^{\prime}-s_{1}}{s_{1}-s_{3}} \chi\left(s_{2}, s_{1} ; s_{1}^{\prime}, \boldsymbol{x} ; \boldsymbol{y}\right)+\frac{s_{1}^{\prime}-s_{2}}{s_{3}-s_{2}} \chi\left(s_{2}, s_{1} ; s_{3}, \boldsymbol{x} ; \boldsymbol{y}\right) \\
& +\left(s_{1}^{\prime}-s_{3}\right) \chi\left(s_{2}, s_{3} ; s_{1}^{\prime}, \boldsymbol{x} ; \boldsymbol{y}\right) \chi\left(s_{3}, s_{1} ; s_{3}, \boldsymbol{x} ; \boldsymbol{y}\right)=0 .
\end{aligned}
$$

We notice that a similar equation in terms of the usual KP coordinates $t_{n}$ has been derived in [21]. By taking the limit $s_{1}^{\prime} \longrightarrow s_{3}$ on this expression it follows

$$
\left(\frac{\partial}{\partial s_{3}}-\frac{s_{2}-s_{1}}{\left(s_{1}-s_{3}\right)\left(s_{2}-s_{3}\right)}\right) \chi\left(s_{2}, s_{1} ; s_{3}, \boldsymbol{x} ; \boldsymbol{y}\right)=\chi\left(s_{2}, s_{3} ; s_{3}, \boldsymbol{x} ; \boldsymbol{y}\right) \chi\left(s_{3}, s_{1} ; s_{3}, \boldsymbol{x} ; \boldsymbol{y}\right) \text {. }
$$

Notice that this means that for any three different $i, j, k$ we have

$$
\left(\frac{\partial}{\partial x_{i}}-\frac{x_{j}-x_{k}}{\left(x_{k}-x_{i}\right)\left(x_{j}-x_{i}\right)}\right) \chi\left(x_{j}, x_{k} ; \boldsymbol{x} ; \boldsymbol{y}\right)=\chi\left(x_{j}, x_{i} ; \boldsymbol{x} ; \boldsymbol{y}\right) \chi\left(x_{i}, x_{k} ; \boldsymbol{x} ; \boldsymbol{y}\right) .
$$

Thus, it follows at once that

$$
\beta_{i j}(\boldsymbol{x} ; \boldsymbol{y}):=\psi\left(x_{i}, x_{j} ; \boldsymbol{x} ; \boldsymbol{y}\right)=\lim _{\epsilon \rightarrow 0} \psi\left(x_{i}(1+\epsilon), x_{j}(1+\epsilon) ; \boldsymbol{x} ; \boldsymbol{y}\right),
$$

satisfy the system of Darboux equations for an infinite-dimensional conjugate net 23

$$
\frac{\partial \beta_{j k}}{\partial x_{i}}=\beta_{j i} \beta_{i k}
$$


For example if we take $\tau \equiv 0$, then $\psi=\psi_{0}$ and we get the following solution of the Darboux equations

$$
\beta_{i j}(\boldsymbol{x} ; \boldsymbol{y})=\frac{1}{x_{i}-x_{j}} \frac{\prod_{n \neq j}\left(1-x_{n} / x_{j}\right)}{\prod_{n \neq i}\left(1-x_{n} / x_{i}\right)} \prod_{n \geq 1} \frac{1-y_{n} / x_{i}}{1-y_{n} / x_{j}} .
$$

Analogously, by taking into account that

$$
\tau=\tau(\boldsymbol{x} ; \boldsymbol{y}) \rightarrow \tilde{\tau}(\boldsymbol{x} ; \boldsymbol{y}):=\tau(\boldsymbol{y} ; \boldsymbol{x}),
$$

is a symmetry of (11), it can be proved that the functions

$$
\tilde{\beta}_{i j}(\boldsymbol{x} ; \boldsymbol{y}):=-\psi\left(y_{j}, y_{i} ; \boldsymbol{x} ; \boldsymbol{y}\right),
$$

satisfy the Darboux system

$$
\frac{\partial \tilde{\beta}_{j k}}{\partial y_{i}}=\tilde{\beta}_{j i} \tilde{\beta}_{i k}
$$

The fact that the Darboux system is associated with the one-component KP hierarchy has been already mentioned in [21] within a different approach.

We finally indicate how to get from the KP hierarchy in continuous Miwa variables to the discrete KP hierarchy. To this end, it is enough to constraint all the continuous Miwa coordinates $\left(x_{i}, y_{i}\right),(i \geq 1)$ to take values on a fixed finite set $\left\{a_{1}, \ldots, a_{N}\right\},(N \geq 2)$. In this way, a set of discrete Miwa variables $\left(l_{1}, \ldots, l_{N}\right)$ can be introduced by

$$
\boldsymbol{t}=\sum_{i=1}^{\infty}\left(\left[x_{i}\right]-\left[y_{i}\right]\right)=\sum_{n=1}^{N} l_{n}\left[a_{n}\right]
$$

Hence functions $\alpha(\boldsymbol{x} ; \boldsymbol{y})$ become functions depending on the discrete variables $l_{i}$. If we now consider (30) with $\boldsymbol{s}=\left(a_{1}, \ldots, a_{N}\right), \boldsymbol{s}^{\prime}=0$, then we get

$$
\left|\begin{array}{ccccc}
1 & a_{1} & \ldots & a_{1}^{N-2} & a_{1}^{N-2} \tau_{1} \hat{\tau}_{1} \\
1 & a_{2} & \ldots & a_{2}^{N-2} & a_{2}^{N-2} \tau_{2} \hat{\tau}_{2} \\
\ldots & \ldots & \ldots & \ldots & \ldots \\
\ldots & \ldots & \ldots & \ldots & \ldots \\
1 & a_{N} & \ldots & a_{N}^{N-2} & a_{N}^{N-2} \tau_{N} \hat{\tau}_{N}
\end{array}\right|=0
$$

where

$$
\begin{aligned}
\hat{\tau}_{i} & :=\tau\left(l_{1}+1, \ldots, l_{i-1}+1, l_{i}, l_{i+1}+1, \ldots, l_{N}+1\right), \\
\tau_{i} & :=\tau\left(l_{1}, \ldots, l_{i-1}, l_{i}+1, l_{i+1}, \ldots, l_{N}\right) .
\end{aligned}
$$

These equations constitute the discrete KP hierarchy [22]. 


\section{References}

[1] B.B. Kadomtsev and V.I. Petviashvili, Sov. Phys. Doklady, 15, 539, 1970.

[2] M. J. Ablowitz and P. A. Clarkson, Solitons, nonlinear evolution equations and inverse scattering (Cambridge Univ. Press, Cambridge, 1991).

[3] B. G. Konopelchenko, Introduction to multidimensional integrable equations (Plenum Press, London, 1992)

[4] T. Shiota, Inven. Math. 83, 333, 1986.

[5] R. Dijkgraaf, Intersection theory, integrable hierarchies and topological field theory in New symmetry principles quantum field theory, Nato ASI, Cargese 1991 (Plenum Press, London, 1991)

[6] A. Morozov, Phys. Usp. 37, 1, 1994.

[7] P. Di Francesco, P. Ginsparg and J. Zinn-Justin, Phys. Rep. 254, 1, 1995.

[8] M. Sato and V. Sato, RIMS Kokyuroku, 439, 30, 1981.

[9] E. Date, M. Jimbo, M. Kashiwara and T. Miwa, Transformation Groups for Soliton Equations in Nonlinear Integrable Systems-Classical Theory and Quantum Theory, edited by M. Jimbo and T. Miwa (World Scientific, Singapore, 1983).

[10] M. Jimbo and T. Miwa, Solitons and Infinite Dimensional Lie Algebras, Publ. RIMS Kyoto University, 19, 943, 1983.

[11] T. Miwa, Proc. J. Acad., 58, Ser. A,9, 1982.

[12] S. Saito, Phys. Rev. Lett, 59, 1798, 1987.

[13] I. Krichever et al, Commun. Math. Phys., 188, 267, 1997.

[14] H. Weyl, The classical groups (Princeton Univ. Press, Princeton, 1939).

[15] I. G. Macdonald, Symmetric functions and Hall polynomials (Clarendon Press, Oxford, 1979). 
[16] G. Segal and G. Wilson, Loop Groups and Equations of KdV Type, Publ. Math. I. H. E. S. 61, 5, 1985.

[17] A. Pressley and G. Segal, Loop Groups (Oxford University Press, Oxford, 1986).

[18] M. Adler and P. Van Moerbeke, Adv. in Math. 108, 140, 1994.

[19] P. G. Grinevich and A. Yu. Orlov, Flag spaces in KP theory and Virasoro action on det $D_{j}$ and Segal-Wilson $\tau$-function, [in:] Problems in modern QFT A. A. Belavin, A. U. Klimyc and A. B. Zamolodchikov (Eds.), pp. 86-106 (Springer, Berlin, 1989)

[20] A. Zabrodin, A survey of Hirota's difference equations, preprint olvint/9704001, 1997.

[21] L. V. Bogdanov and B. G. Konopelchenko, J. Math. Phys.39, 4683, 1998.

[22] Y. Otha, R. Hirota, S. Tsujimoto and T. Imai, J. Phys. Soc. Japan, 62, No. 6, 1872, 1993.

[23] G. Darboux, Lecons sur les systemes orthogonaux et les coordonnes curvlignes (Paris, 1897). 